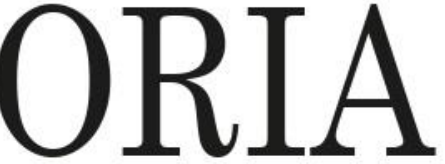

ISSN: 2027-5137

Enero - Junio, Año 2021 - Tunja, Colombia

Conflicto armado en Guatemala: reconstrucción histórica y memoria colectiva del pueblo maya chuj

https:/doi.org/10.19053/20275137.n22.2021.10791

Fabiola Mayari López Bracamonte Páginas 323-357

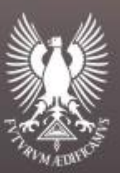




\title{
Conflicto armado en Guatemala: reconstrucción histórica y memoria colectiva del pueblo maya chuj"
}

\author{
Fabiola Manyari López Bracamonte ${ }^{1}$ \\ CIMSUR-UNAM, México
}

Recepción: 25/03/2020

Evaluación: 15/07/2020

Aprobación: 26/09/2020

Artículo de Investigación e Innovación

https:/doi.org/10.19053/20275137.n22.2021.10791

\section{Resumen}

A principios de 1980 se desencadenó una de las más intensas y atroces represiones militares en la larga historia de violencia política de Guatemala. En el marco de las disputas de la Guerra fría los pueblos mayas se volvieron enemigos internos para los proyectos nacionales, por lo que las estrategias de terror se ensañaron con ellos, obligándoles a desplazarse para resguardar su vida física y cultural. Aunque la violencia de Estado marcó una ruptura, experiencias como las del pueblo maya chuj se erigen como ejemplo de resistencia física y simbólica. Con el objetivo de aportar nuevos datos de análisis y reflexión en torno a la violencia ejercida sobre los pueblos mayas en Guatemala, este artículo realiza una reconstrucción histórica de esos acontecimientos retomando aspectos de la

\footnotetext{
* Resultados parciales de la investigación doctoral Resiliencia comunitaria del pueblo maya-chuj: conocimientos culturales, capacidades sociales y estrategias organizativas. El Colegio de la Frontera Sur, México.

1 Doctora en Ciencias en Ecología y Desarrollo Sustentable, postdoctorante en el Centro de Investigaciones Multidisciplinarias de Chiapas y la Frontera Sur, Universidad Nacional Autónoma de México. Otras publicaciones: Fabiola Manyari López Bracamonte y Fernando Limón Aguirre, "Componentes del proceso de resiliencia comunitaria: conocimientos culturales, capacidades sociales y estrategias organizativas,» PSICIENCIA, Revista Latinoamericana de ciencia psicológica Vol. 9, $\mathrm{n}^{\circ} 3 . \otimes$ manyaril@hotmail.com (ㄴ) https://orcid.org/0000-0002-0754-9480.
} 
memoria histórica y la memoria colectiva del pueblo maya chuj. Para ello se analizan la cualidad de las interacciones políticas, sociales y culturales de los aspectos esenciales que desencadenaron, acompañaron y marcaron el contexto del conflicto armado, el desplazamiento forzado, el resguardo y el refugio en México, así como el retorno y repatriación a Guatemala del pueblo chuj.

Palabras clave: guerra fría, desplazamiento forzado, violencia de Estado, enemigos internos, refugio, retorno.

\title{
Armed conflict in Guatemala: historical reconstruction and collective memory of the Mayan Chuj people
}

\begin{abstract}
At the beginning of 1980, one of the most intense and atrocious military repressions in the long history of political violence of Guatemala was unleashed. Within the framework of the disputes of the Cold War, the Mayan peoples became internal enemies of national projects and, therefore, they were the targets of strategies of terror, forcing them to migrate in order to protect their physical and cultural lives. Although the state violence marked a rupture, experiences such as those of the Mayan Chuj people are an example of physical and symbolic resistance. With the aim of contributing new data for analysis and reflection concerning the violence exerted over the Mayan peoples in Guatemala, this article conducts a historical reconstruction of those events, revisiting aspects of the historical and collective memory of the Mayan Chuj people. To this end, the quality of the political, social and cultural interactions of the essential aspects that unleashed, accompanied and marked the context of the armed conflict, the forced displacement, protection and refuge in Mexico are analysed, as well as the return and repatriation of the Mayan Chuj people to Guatemala.
\end{abstract}

Key words: Cold War, forced displacement, state violence, internal enemies, sanctuary, return. 


\section{Le conflit armé au Guatemala: reconstruction historique et mémoire collective du peuple maya chuj}

\section{Résumé}

$\mathrm{Au}$ début de 1980, une atroce répression militaire s'est déclenché au Guatemala, un pays qui a connu une longue histoire de violence politique. Dans le cadre des disputes propres à la Guerre froide, les peuples mayas sont devenus ennemis internes des projets nationaux, et c'est pourquoi les stratégies de terreur les ont pris pour cible les obligeant à se déraciner pour sauver leurs vies et leurs cultures. Si bien que la violence de l'État a marqué une rupture, des expériences comme celle du peuple maya chuj s'érigent comme un exemple de résistance physique et symbolique. Dans le dessein d'apporter des nouvelles données pour l'analyse sur la violence exercée contre les peuples mayas au Guatemala, cet article propose une reconstruction historique de ces événements historiques en revenant sur la mémoire historique et la mémoire collective du peuple maya chuj. Pour ce faire, on analyse la qualité des interactions politiques, sociales et culturelles des situations ayant marqué le contexte du conflit armé, le déplacement forcé, la migration au Mexique et le retour du peuple chuj au Guatemala.

Mots-clés: Guerre froide, déplacement forcé, violence d'État, ennemis internes, exil, retour.

\section{Introducción}

Durante todo el siglo XX, pero en particular, en sus últimos treinta años, la vida política y social de Guatemala estuvo marcada por períodos de grandes pugnas que derivaron en una confrontación de diferentes grupos de interés, caracterizada por el intervencionismo extranjero y el uso desmedido de la fuerza del Estado a través de su brazo militarizado contra movimientos populares, insurgentes y población civil.

Recrudecido a principios de 1980, el Ejército guatemalteco perpetró una serie de matanzas en forma de masacres 
indiscriminadas que desplegaron su mayor crueldad contra los pueblos mayas. Viéndose forzados a desplazarse fuera de Guatemala, permanecieron refugiados en México alrededor de quince años para salvaguardar sus vidas y su legado histórico. Con la Firma de los Acuerdos de Paz en 1996, miles de familias encontraron las garantías para un retornar masivamente a Guatemala.

Esos acontecimientos involucran dos escalas: las fuerzas globales y locales que, motivaron las alianzas políticas y estrategias bélicas, y las experiencias incorporadas que contienen emociones, reflexiones e impactos en la vida individual y social. En este artículo se busca reconstruir los sucesos de ambas escalas, concatenando las dimensiones históricas y experienciales, con el objetivo de aportar nuevos datos de análisis y reflexión en torno a la violencia ejercida sobre los pueblos mayas en Guatemala.

Para la comprensión histórica de los sucesos que dieron pie a la violencia de Estado y el desplazamiento forzado, se realiza una descripción densa de los componentes geopolíticos que intercedieron en la gesta y desarrollo del conflicto armado en Guatemala, procurando articular la escala global y la local. La dimensión experiencial se aborda desde los relatos, testimonios y reflexiones que son parte de la memoria colectiva del pueblo maya-chuj ${ }^{2}$, en torno a lo acontecido durante el conflicto, el desplazamiento y el refugio en México.

La recolección de datos se realizó a partir de una investigación documental bibliográfica, hemerográfica y de archivos escritos y audiovisuales. La memoria colectiva es abordada a partir del trabajo etnográfico durante cuatro años (2015-2018), realizado en el marco de la investigación doctoral sobre resiliencia comunitaria del pueblo maya-chuj ${ }^{3}$. El trabajo de campo se sustentó en la observación directa, y entrevistas

2 El pueblo Maya-chuj constituye un grupo maya localizado actualmente entre la frontera de Guatemala y el sur de México. En el lado guatemalteco ocupa tres municipios en el departamento de Huehuetenango: San Mateo Ixtatán, San Sebastián Coatán y Nentón, en el lado mexicano principalmente en el municipio de la Trinitaria, Chiapas.

3 Investigación doctoral Componentes del proceso de resiliencia comunitaria: conocimientos culturales, capacidades sociales y estrategias organizativas (2018). 
semiestructuradas individuales y colectivas en aldeas del municipio de Nentón, Huehuetenango en Guatemala, y en colonias del municipio de la Trinitaria en México.

La temporalidad se enmarca entre 1970 y 2018. La descripción-problematización se agrupa en cinco períodos: 1) Antecedentes y generalidades del conflicto armado en Guatemala (1970-1981); 2) Violencia de Estado y el desplazamiento forzado en territorio maya chuj (1981-1983); 3) Permanencia y refugio en Chiapas, México del pueblo maya chuj (1983-1996); 4) Acontecimientos en Guatemala durante el período del Refugio (1983-1996); y 5) Retorno y repatriación hacia Guatemala del pueblo maya chuj (1996-2016).

\section{Antecedentes y generalidades del conflicto armado en Guatemala (1970-1981)}

Escenario global

Guatemala ha sido escenario de diferentes tensiones y enfrentamientos políticos desde su conformación como República en 1847. A lo largo de su historia sociopolítica, se ha enfrentado a constantes regímenes dictatoriales, así como a movilizaciones insurgentes, entre ellas la revolución desencadenada en 1944 con diez años de duración, el derrocamiento de diferentes gobiernos, y el conflicto armado iniciado en 1960 y sostenido por más de treinta años.

Todo ello influenciado por intereses provenientes principalmente de la política exterior de gobiernos de Estados Unidos de América (EEUU) ${ }^{4}$, que desde un siglo atrás habían legitimado el intervencionismo como parte de la Doctrina Monroe, y que en medio de las tensiones de la postguerra, alertados por la victoria cubana, recrudecieron en

Doctorado en Ciencias en Ecología y Desarrollo Sustentable, El Colegio de la Frontera Sur, México, Consejo Nacional de Ciencia y Tecnología (CONACYT), México.

4 Raúl Sohr, Centroamérica en guerra. Las fuerzas armadas en de Centroamérica y México (México: Alianza Editorial Mexicana, 1989), 30-36. 
Latinoamérica sus estrategias de contención para no perder el dominio comercial y político ${ }^{5}$.

A principios de los años 80 del siglo pasado, la Guerra Fría en América Latina alcanzó un nuevo auge, especialmente en Centroamérica. La intervención política y militar norteamericana se volvió mucho más directa y abierta de lo que había sido en otras épocas ${ }^{6}$.

En Guatemala bajo el gobierno del militar y terrateniente Fernando Lucas García (1978-1982), se brindó apoyo para la implementación de la Doctrina de Seguridad Nacional ${ }^{7}$. Esta visión de seguridad a partir de la militarización instauraba la noción de "enemigo interno» que construía una "otredad adversaria». En esta no solo entraban grupos guerrilleros señalados como comunistas, sino cualquier grupo que tuviera ideas opuestas a las de los proyectos nacionales y sus gobiernos militares ${ }^{8}$.

Esa visión legitimó la intervención militar violenta en diferentes zonas del país, sobre todo en el noreste habitado por poblaciones indígenas desarmadas y ajenas al conflicto ${ }^{9}$. Documentos de reconstrucción de la memoria histórica, análisis académicos de los acontecimientos de esa época e incluso

5 Patrick Ball, Paul Kobrak, y Herbert Spirer, Violencia institucional en Guatemala, 1960 a 1996: Una reflexión cuantitativa (Estados Unidos de América: American Association for the Advancement of Science (AAAS) Science and Human RightsProgram. Centro Internacional para Investigaciones en Derechos Humanos (CIIDH), 1999), 16-18.

6 Edelberto Torres, "Guatemala: desarrollo, democracia y los acuerdos de paz", Revista Centroamericana de Ciencias Vol. 3, nº 2 (2006): 11-48.

7 Francisco Leal «La Doctrina de Seguridad Nacional en América Latina: materialización de la guerra fría en América del Sur,» Revista de Estudios Sociales, $\mathrm{n}^{\circ} 15$ (2003): 74-87.

8 Alexandra Pita, Enrique Coraza y Amador Karla, «La Doctrina de Seguridad Nacional en América Latina: el concepto de enemigo,» en La Guerra Fría y las Américas, coord. Avital Bloch y María del Rosario Rodríguez (México: Universidad de Colima-Centro Universitario de Investigaciones Sociales- Universidad Michoacana de San Nicolás de Hidalgo- Instituto de Investigaciones Históricas, 2013), 16-34.

9 CEH (Comisión para el Esclarecimiento Histórico), Informe de la Comisión para el Esclarecimiento Histórico. TOMO III: Las violaciones de los derechos humanos y los hechos de violencia (Guatemala: Oficina de Servicios para Proyectos de las Naciones Unidas (UNOPS), 1999), 29. 
reflexiones religiosas, han concluido y denunciado que más que acabar con los grupos armados "peligrosos» ${ }^{10}$, el propósito de las intervenciones, se centró en generar condiciones favorables para que el Estado entrara en la lógica moderna de sus aliados comerciales -EEUU principalmente- ${ }^{11}$.

Es decir, que el gobierno guatemalteco constituido por antiguos grupos oligarcas y financiados por capitales extranjeros, usó la fuerza militar para implementar el modelo moderno de sobreexplotación de materias primas, y en su momento, el consumo masivo de bienes y servicios dentro el libre mercado ${ }^{12}$. Motivado por los beneficios económicos para sus grupos de poder y cargado de prejuicios racistas, arremetió contra los pueblos indígenas y sus organizaciones culturales «atrasadas», para en su lugar implementar proyectos agroindustriales en sus territorios ${ }^{13}$. La producción cultural indígena de conocimiento en el manejo de la tierra, se transformó en un producto para el mercado, pero ya desprovista de su connotación simbólica de relación con la misma ${ }^{14}$.

Lo anterior se materializó en la ejecución de acciones de sometimiento e imposición deliberadas por organismos dependientes del Estado ${ }^{15}$-Violencia de Estado-, para

10 Universidad de San Carlos de Guatemala, Guatemala: Proceso de paz y perspectivas. Escuela de historia. S.f. 2-3.

11 Majawil Q'ij, El Nuevo Amanecer (Guatemala: Folleto informativo de la Coordinación maya MajawilQ'ij", 1992), 1-7; Reflexión Cristiana en Guatemala, Derechos de los pobres, derechos de dios, (Guatemala: grupo de Reflexión Cristiana en Guatemala, noviembre 1990), 25-28; Francisco Lazo, «La estrategia norteamericana y la geopolítica de América Central en América Central y el Caribe,» en Zona de Conflictos y problemas geopolíticos (México: Cuaderno de Trabajo $\mathrm{N}^{\circ} 8$. Centro de Investigación y Trabajo social, 1986), 23-42.

12 Friedrich Katz. "La guerra fría en América Latina,» en Espejos de la guerra fría: México, América Central y el Caribe, coord. Daniela Spencer (México: Centro de Investigaciones y Estudios Superiores en Antropología Social. Porrúa, 2004), 11-30.

13 Gabriel Aguilera y Jorge Imery, Dialéctica del terror en Guatemala (Costa Rica: Universitaria Centroamericana, 1981), 47.

14 Centro de Estudios Integrados de Desarrollo Comunal Guatemala polos de desarrollo. El caso de la desestructuración de las comunidades indígenas (México: Praxis, 1988), 35 .

15 Arzobispado de Guatemala y Oficina de Derechos Humanos, "Versión popular del informe REMHI" (Guatemala: Nunca Más. Oficina de Derechos Humanos del Arzobispado de Guatemala, 2000), 6-12. 
profundizarlas directrices del capitalismo a través de la transformación de los códigos simbólicos contenidos en la identidad étnica ${ }^{16}$. De ese modo se trasladó el conflicto social hacia áreas rurales, involucrando a las comunidades mayas del altiplano occidental.

La cúspide de esa violencia se dio entre 1982 y 1983, durante el gobierno golpista de Efraín Ríos Montt ${ }^{17}$. Se calcula que en ese período más de 250,000 personas fueron asesinadas, más de 400 aldeas fueron masacradas, y más de millón y medio de personas se desplazaron forzadamente ${ }^{18}$.

Cabe mencionar que estos componentes también estaban presentes en las experiencias de otros países de Latinoamérica. Como en Guatemala, el intervencionismo extranjero coludido con el poder militar generó procesos transnacionales de formación ideológica y técnica contra el expansionismo comunista que, al combinarse con movimientos locales, desembocaron en una desproporcionada represión de grupos insurgentes y en la vejación de la sociedad civil ${ }^{19}$.

Todo lo descrito se combinó con la impunidad jurídica, el cierre de espacios para la participación social y de la libertad de expresión ${ }^{20}$. Además se integraron estrategias de

16 Centro de Estudios Integrados de Desarrollo Comunal, Guatemala polos de desarrollo, 173; César Castañeda, Lucha por la tierra, retornados y medio ambiente en Huehuetenango (Guatemala: FLACSO, 1998), 101-103.

17 Carlos Figueroa, «Genocidio y terrorismo de Estado en Guatemala (1954-1996): una interpretación,» en Guatemala: historia reciente (1954-1996). Tomo I: proceso político y antagonismo social (Guatemala: Facultad Latinoamericana de Ciencias Sociales, 2012), 68-89; Sergio Palencia-Ferrer, «Rebelión social y contrainsurgencia en Guatemala, 1981-1983. Conformación estatal y potencialidad revolucionaria,» Liminar. Estudios Sociales y Humanísticos Vol. XII, n 1 (2014): 161-176.

18 Paul Kobrak, Huehuetenango. Historia de una guerra (Guatemala: Centro de Estudios y Documentación de la Frontera Occidental de Guatemala (CEDFOG), 2003), 33-35; Beatriz Manz, Guatemala. Cambios en la comunidad, desplazamiento y repatriación (México: Escuela de Antropología Wellesley College, Centro de Estudios Internacionales Harvard University. Traducción libre al español de la primera versión del documento en inglés; no revisada por la autora. Praxis, 1986), 17.

19 Ariel Armony, «Trasnacionalización del Caribe: catalizador de la proyección soviética en América Latina,» en Espejos de la Guerra Fría: México, América Central y el Caribe, coord. Daniela Spencer (México: Centro de Investigaciones y Estudios Superiores en Antropología Social. Porrúa, 2004), 319-330.

$20 \mathrm{CEH}$, Informe de la Comisión para el Esclarecimiento Histórico, 98-103. 
sometimiento simbólico mediante acciones de intimidación y terror, así como de adoctrinamiento religioso mediante la expansión de religiones de corrientes teológicas cristianas protestantes (evangélicas y pentecostales) ${ }^{21}$.

Con el fin de contravenir prácticas culturales relacionadas con la espiritualidad maya que favorecían la cohesión comunitaria, y para menguar el control político-religioso de las autoridades tradicionales como el Alcalde Rezador o Los Principales ${ }^{22}$. Por su parte, la iglesia católica participó a través de discrepancias entre las posturas conservadoras y progresistas, apoyada en la «teología de la liberación», que originó algunas afinidades al movimiento guerrillero, especialmente, notorias en parroquias localizadas en las montañas del noroccidente del país ${ }^{23}$.

\section{Respuesta insurgente}

De 1954 a 1980 la economía guatemalteca experimentó un crecimiento económico importante, de los mayores en toda América Latina, derivado en su mayor parte por la inversión norteamericana para la producción de nuevos cultivos de exportación, además del café, caña de azúcar, algodón, ganadería y cardamomo ${ }^{24}$; sin embargo, ese crecimiento se caracterizó por un aumento de las desigualdades sociales y los niveles de pobreza. El $20 \%$ más rico de la población captaba casi el $60 \%$ del ingreso, mientras que el $20 \%$ más pobre apenas alcanzaba a percibir el $3 \%{ }^{25}$. Esto aunado a la historia de explotación, marginación e injusticia social, propició movimientos sociales que pugnaban por mejorar las condiciones de vida de la población en términos más equitativos.

21 Kobrak, Huehuetenango: historia de una guerra, 42.

22 Centro de Estudios Integrados de Desarrollo Comunal Guatemala polos de desarrollo, 35 .

23 Héctor Gramajo, La difícil transición política en Guatemala. De la guerra... a la guerra (Guatemala: Fondo de Cultura Editorial, 1995), 121-122.

24 Historia del pueblo de Guatemala. Documento informativo de distribución anónima, 88.

25 CEPAL «Notas sobre la evolución del desarrollo social del istmo centroamericano hasta 1980». (Naciones Unidas. Consejo Económico y Social. Distribución limitada, E/CEPAL/MEX/1982/L.2 6/Rev.1, 1982, acceso el 29 de marzo 03 de 2017 , http://repositorio.cepal.org/bitstream/handle/11362/26485/S8200536_ es.pdf? sequence $=1 \&$ isAllowed $=y$. 
En un primer momento, alrededor de 1970, los movimientos se constituyeron en amplias luchas populares, urbanas y rurales, y en huelgas magisteriales que apostaban por una lucha legal, desde los sindicatos y partidos políticos ${ }^{26}$. Estos brotes fueron fuertemente reprimidos gracias a la imperante fuerza militar, y a la estrategia de silenciar impunemente cualquier forma de resistencia u oposición al poder hegemónico establecido.

Frente a la derrota, a finales de la misma década, las demandas insurgentes tuvieron un proceso reorganizativo en el que se integró una lucha armada, y afianzaron sus bases de operaciones en las montañas del altiplano occidental. En ese segundo momento, influenciados por la experiencia cubana, y con su apoyo político, logístico, de instrucción y entrenamiento, surgieron en la selva norte de Quiché, agrupaciones armadas como el Ejército Guerrillero de los Pobres (EGP) ${ }^{27}$, y la Organización del Pueblo en Armas (ORPA), que inició operaciones en la Boca Costa de San Marcos ${ }^{28}$.

También, se crearon otros organismos políticos como el Comité de Unidad Campesina (CUC), y el Comité Guatemalteco de Unidad Patriótica (CGUP). El primero, un grupo multiétnico cuya organización estaba dirigida por indígenas en el occidente del país; el segundo, un frente político encargado de la realización de reuniones y foros de denuncia de la represión estatal que se vivía ${ }^{29}$. En febrero de 1982 surgió la Unidad Revolucionaria Nacional Guatemalteca (URNG) como aglutinadora de las organizaciones guerrilleras existentes $^{30}$.

26 Javier Gurriaran, La resistencia en Guatemala (México: Nuestro Tiempo, 1989), 17-21.

27 Mario Tejada, Historia social del norte de Huehuetenango (Guatemala: Magna Torres, 2002), 169.

28 SonjaPerkič-Krempl, «El Derecho a Saber» (Tesis de Maestría, Universidad Rafael Landivar, 2006), 23.

29 Figueroa. «Genocidio y terrorismo de Estado en Guatemala (1954-1996): una interpretación,» 68-89.

30 Centro de Noticias de Guatemala, Guatemala 1986-1987. Crisis del 1er. año de Gobierno de Vinicio Cerezo (EEUU: documento anónimo, 1987), 6. 
En una desproporcionada contraofensiva, miles de soldados condujeron el conflicto de manera sistemática por todo el altiplano ${ }^{31}$, no solo contra las unidades guerrilleras, sino sobre todo, contra cientos de aldeas de pueblos originarios que nunca estuvieron armados. Con el objetivo de vigilar y controlar la zona, incluyendo caminos y sociedad civil, en el que la insurgencia se movilizaba, el Ejército guatemalteco instaló bases militares en las cabeceras municipales y aldeas dentro de los departamentos de Huehuetenango y Quiché, ambos con mayoritaria población indígena ${ }^{32}$.

\section{Violencia de Estado y desplazamiento forzado en el territorio maya chuj (1981-1983).}

En el departamento de Huehuetenango, sobre todo en los municipios con presencia chuj como San Mateo Ixtatán y Nentón, desde 1960 y hasta antes del desplazamiento forzado de 1982, la tensión entre mestizos e indígenas iba en aumento debido a las disputas por la implementación de proyectos agroindustriales y forestales en territorios indígenas. Los reclamos por condiciones laborales y salariales más justas, y a la ampliación de espacios de poder y decisión de líderes indígenas cobraban más fuerza.

Un punto cúspide de estas tensiones se dio a finales de la década de los años 1970, cuando grupos chuj junto con autoridades locales se enfrentaron a la empresa nicaragüense Cuchumaderas, que ganó una demanda para el desarrollo de proyectos forestales que había iniciado en la zona. Al mismo tiempo, otro sector demandaba derechos laborales en una mina de sal negra, próxima a la aldea Petanac y Guaisná, en San Mateo Ixtatán ${ }^{33}$.

31 David Stoll, Between Two Armies in the Ixil Towns of Guatemala (Nueva York: Columbia University Press, 1993), 101-106.

32 Kobrak 2001 en Tejada, Historia social del norte de Huehuetenango, 157-158; Davis Shelton, «Introduction: Sowing the Seeds of Violence,» en Harverst of violence: The Maya Indians and the Guatemala Crisis, ed. Robert M. Carmarck (EEUU: University of Oklahoma Press-Norman, 1988), 3-16.

33 Luis Solano, Contextualización Histórica de la Franja Transversal del Norte (FTN) (Guatemala: Centro de Estudios y Documentación de la Frontera Occidental de Guatemala CEDFOG, 2012), 25-26. 
Esas afrentas incomodaron a los dueños de los capitales de las empresas instaladas en la región, por lo que se incrementó la urgencia de controlar el territorio sometiendo a su población; actividades que fueron respaldadas por el gobierno nacional. Por otro lado, esos acontecimientos generaron puntos de interlocución entre grupos organizados chuj y los planteamientos guerrilleros que promovían, entre otras cosas, la justicia social y los derechos agrarios.

Aunque esas afinidades no se dieron de manera homogénea en la población, ni en aquellas más alejadas del centro político que representaban las cabeceras municipales. La versión oficial de los mandos militares aludió a que esa relación era generalizada y requería de la incursión y represión del Ejército en toda la región ${ }^{34}$. Como ya se ha mencionado, versiones no oficiales señalaban que esa intervención, aunque se justificaba en la lucha insurgente, pretendía la implementación de un proyecto económico mediante la apropiación del territorio y de sus pobladores.

Habitantes chuj de la aldea de Yalanhbojoch, ubicada en Nentón, Huehuetenango, recuerdan el contacto con la guerrilla:

La guerrilla anduvo primero haciendo sus discursos como era la idea que tienen, entonces la gente también la entendió, ¿porqué estamos mal?, ¿porqué estamos hasta aquí?, si nuestros antepasados, nuestros abuelos que vivieron antes vivieron en lugares más fértiles, son más mejores que los lugares de aquí, pero con la idea de los terratenientes y como esto son extranjeros que vinieron, no son nativos del continente. Entonces algunos muchachos que empezaron a participar en su discurso, algunos ya al final si se estaban interesando de colaborar con ellos, pero en eso llegó el Ejército y dijeron que no se podía, y ya la guerrilla empezó a pasar menos y los muchachos ya no se unieron más. También con el Ejército aquí ya desapareció este grupo que se les llamó el CCN (Comité Clandestino Local) que era el encargado de juntar las colaboraciones para darle a la guerrilla ${ }^{35}$.

34 Tejada, Historia social del norte de Huehuetenango, 177.

35 Miguel (habitante de la aldea de Yalanhb'ojoch), entrevista por Fabiola Manyari López Bracamonte, 18 de enero de 2018. 
Este relato tuvo lugar en 1980, cuando grupos guerrilleros recorrían las aldeas buscando nuevos aliados. Como claramente menciona, el discurso de la guerrilla tuvo impacto en algunos habitantes, sobre todo en los más jóvenes pero eso involucró a todos los habitantes, y el vínculo fue inmediatamente sofocado con la llegada del Ejército. Esto revela que si bien había contactos con la guerrilla, estos no estaban coludidos con sus estrategias armadas, argumento que posteriormente el gobierno guatemalteco usó para justificar la intervención armada y violenta del Ejército.

Otra persona de la misma aldea recuerda el primer contacto con el Ejército de la siguiente manera:

La primera vez que vinieron [el Ejército] solo nos convocaron a una reunión donde nos platicó sobre proyectos de agua potable, construcción de carreteras, crédito del banco Bandesa y fertilizantes [junio 1982]. En ese tiempo dejaron organizadas las Patrullas de Autodefensa Civil que se quedaron encargados de vigilar la aldea con palos puntiagudos y de ahí regresaron a la misma dirección donde vinieron ${ }^{36}$.

Aunque en esta primera visita el Ejército fue con una actitud aparentemente de ayuda a los aldeanos, en menos de un mes, entre julio y agosto de 1982, como parte de las operaciones denominadas Zacualpa, el Ejército nuevamente se hizo presente en la zona, pero ya con una estrategia de aniquilación ${ }^{37}$. Esos acontecimientos quedaron marcados en la memoria de los habitantes de las aldeas. Un testigo cercano lo describe de la siguiente manera:

Se fueron a San Francisco [el Ejército] y ya eran como las tres de la tarde y empezaron el ruido de las bombas, fue cuando empezó la masacre el mismo 17 de julio [de 1982]. Entonces como estamos patrullando [en las PACs] por todos lados, y nosotros estábamos ahí exactamente por el campo de futbol, yo estaba con mi papá y un mi hermano, yo apenas

36 Gustavo (habitante de la aldea de Yalanhb'ojoch), entrevista por Fabiola Manyari López Bracamonte, 06 de julio de 2015.

37 Edgar Balsells, Olvido o memoria: el dilema de la sociedad guatemalteca. 2da ed. (Guatemala: FLACSO, 2001), 60-74. 
tenía 14 años y ahí estábamos porque ya nos tomaban en cuenta en esa parte de las patrullas. Yo era pequeño pues, no tenía edad para estar en el servicio pero ya me tocaba ese turno. Ahí estábamos cuando empezó la matanza, y no sabíamos que pasaba porque no podemos ver. Entonces lo que pensamos nosotros era que la guerrilla llegó a enfrentarse ante el Ejército, eso era lo que pensamos, eso comentamos. Se escuchaba las balaceras y el ruido de las bombas que explotaban, muy horrible. Y ya después como dilataron ahí, en la noche como ahí estuvimos toda la noche haciendo vueltas alrededor de la comunidad, ya en la noche se sentía el olor que venía de la gente que estaban quemando. Toda la noche estuvo quemando las casas ${ }^{38}$.

Algunos informes realizados posteriormente señalaron a las cuadrillas del Ejército como «caravanas de la muerte» ${ }^{39}$, pues perpetraron masacres de arrasamiento como la de la finca de San Francisco en Nentón ${ }^{40}$. Las patrullas mencionadas en ambos relatos, se refieren a las Patrullas de Autodefensa Civil (PAC), que consistían en grupos de vigilancia conformada por habitantes de los centros poblados. Concebidas como una manera de consolidar el poder del Ejército sobre las aldeas en disputa en la montaña ${ }^{41}$, movilizaron a los hombres para patrullar los caminos y "resguardar» a los habitantes de la «incursión guerrillera», permitiéndoseles actos de violencia contra sus coterráneos ${ }^{42}$. Según datos oficiales para 1985 existían casi 900,000 patrulleros civiles ${ }^{43}$.

38 Manuel (habitante de la aldea de Yalanhb'ojoch), entrevista por Fabiola Manyari López Bracamonte, 14 de enero de 2018.

39 Arzobispado de Guatemala, Informe del Proyecto Interdiocesano: Recuperación de la Memoria Histórica. TOMO II (Guatemala: Oficina de Derechos Humanos del Arzobispado de Guatemala ODHAG, 1998), 217-220.

40 Contexto Histórico de la Aldea de Yalanhb'ojoch, Contexto Histórico de la Aldea de Yalanhb'ojoch, del municipio de Nentón, Departamento de Huehuetenango (Guatemala: Programa Nacional de Resarcimiento, Unidad de Aplicación de Medidas. Yalanhb'ojoch, 2009), 3.

41 Human Rights Watch/Americas Human Rights in Guatemala Durind President de León Carpio's First Year, trad. Luis E. Bossio (Guatemala: Luna y Sol editorial, 1994), 12.

42 CDHG, Testimonios sobre las Patrullas de Autodefensa Civil (PAC) en Huehuetenango (Guatemala: Comisión de Derechos Humanos de Guatemala,1985), 2; Estela Gutiérrez y Paul Kobrak, Los linchamientos postconflicto y Violencia colectiva en Huehuetenango Guatemala (Guatemala: CEDGOF, 2001), 70-76.

43 CDHG, Testimonios sobre las Patrullas de Autodefensa Civil (PAC), 1. 
Los actos de violencia descritos tuvieron como consecuencia directa que aldeas completas chujes y de otros pueblos mayas huyeran hacia México, y que otros buscaran refugio en localidades cercanas a la cabecera departamental o en la ciudad capital. Muchos de los que se vieron forzados a desplazarse a México buscaron llegar a los sitios de refugio cerca del lago Tziscao en Chiapas, México, territorio ocupado por familiares o conocidos chuj.

Ya nosotros estábamos agarrando el rumbo para México. En el camino vimos tanta gente muerta, mujeres tiradas desnudas, macheteados, niños aplastados en sus cabezas, un gran horror. Nosotros quedamos durmiendo a [en] la frontera mexicana, ahí bajo la lluvia tan fuerte. Ahí organizamos para mandar uno, dos, tres comisiones para ir a buscar nuestro posada, en qué colonia vamos a llegar, si vamos a llegar en km 15 o en Tziscao o en Cuauhtémoc, o a ver dónde llegamos, pero gracias a Dios a los Estados Mexicanos que algunos nos conocen otros no, nos recibieron con brazos abiertos desde ese tiempo en el año 1982. Pero siempre con la pena, con susto terrible también. No llevábamos cosas de alimentación, de principalmente a los niños, a los ancianos, ropa para cambiar, no hay nada ${ }^{44}$.

Horrorizados, con pena, y en esas ínfimas condiciones, miles de personas, incluidas mujeres en avanzado grado de gestación, niños y ancianos huyeron de las masacres. Como lo señala el testimonio, muchas de ellas no lograron llegar a la frontera pues fueron sorprendidas, mutiladas, violadas y asesinadas brutalmente por cuadrillas del Ejército guatemalteco. Las que lograron llegar iniciaron un largo proceso de resistencia física y psicológica, así como de reconstrucción de su vida social.

\section{Refugio del pueblo chuj en Chiapas, México (1983- 1996)}

Guatemala y Chiapas tienen historia en común. Desde hace más de un siglo, cuando se definieron los límites vigentes (año 1882), diferentes pueblos como el chuj, quedaron en una y otra parte de

44 María (habitante de la aldea de Yalanhb'ojoch), entrevista por Fabiola Manyari López Bracamonte, 22 enero 2017. 
la frontera. Desde esas fechas han existido relaciones de trabajo, comerciales, culturales, de parentesco, festividades y ceremonias. Familias y campesinos guatemaltecos repetidamente han cruzado, y cruzan, la frontera mexicana buscando trabajo, para vender sus productos o para visitar a sus familiares y amigos, y al menos en dos ocasiones lo hicieron buscando un lugar seguro para resguardar sus vidas ${ }^{45}$ : en 1954 durante el derrocamiento de Jacobo Arbenz, y en 1980 durante la violencia de Estado ${ }^{46}$.

Este último es el más numeroso en la historia de México, teniendo en cuenta que, tan solo entre 1980 y 1983, más de 100 mil personas guatemaltecas buscaron refugio en México, a causa del incremento de la violencia por las estrategias de arrasamiento $^{47}$. Al llegar a México, los primeros en recibirlos fueron las familias mexicanas con quienes tenían redes de apoyo o parentesco. Les permitieron permanecer en espacios comunales y, posteriormente, algunas familias mexicanas se llevaron a familias guatemaltecas a sus propias casas. Así lo describen algunos testimonios:

Nosotros fuimos a vivir en la Colonia Cuauhtémoc en el municipio de la Trinitaria Chiapas. Los mexicanos al llegar nos recibieron con amor y buscaron lugares públicos donde acomodaron a la gente, una parte en la escuela albergue, otra parte en las casas comunales, y otros en la casa de los vecinos de la colonia mientras designaron un lugar donde nos acampamos todos. Señalaron donde íbamos a cortar hojas para usar como techo y una parte de la montaña para sacar madera para las casitas y para leña. Así lo hicimos,

\footnotetext{
45 Edith Kauffer. "De la frontera política a las fronteras étnicas Refugiados guatemaltecos en México,» Frontera Norte Vol. 17, n 34 (2005): 7-36.

46 Centro de Investigaciones y Estudios Superiores en Antropología Social, Los refugiados Guatemaltecos y lo Derechos Humanos. Cuaderno para Refugiados Guatemaltecos (México: Gobierno del Estado de Chiapas/Consejo Estatal de Fomento a la Investigación y Difusión de la Cultura/DIF-Chiapas/Instituto Chiapaneco de Cultura, 1991), 22.

47 Human RightsWatch/Americas, Human Rights in Guatemala, 53-56; Rosalva Hernández y otros, La experiencia del refugio en Chiapas. Nuevas relaciones en la frontera sur mexicana (México: Academia Mexicana de Derechos Humanos A.C., Centro de Investigaciones y Estudios Superiores en Antropología Social, Consejería en Proyectos para Refugiados Latinoamericanos, OXFAM, United Nations Research Institute for Social Development, Copilco Universidad, 1993), 56.
} 
agradecidos porque nos brindaron un espacio pues más tenemos que respetar ${ }^{48}$.

Adecuar los espacios en los asentamientos a los quellegaban gran cantidad de personas fue una necesidad primordial. Los primeros días de resguardo en territorio mexicano, recurrieron a sus conocimientos de organización social y construcción con materiales naturales para adaptar sitios que les permitieran guarecerse $^{49}$. Posteriormente instituciones mexicanas como el Hospital de Comitán y Diócesis de San Cristóbal de Las Casas, brindaron su ayuda en lo que el gobierno mexicano respondía a la situación. Un tiempo después llegó el Alto Comisionado de las Naciones Unidas para Refugiados (ACNUR), quien dio apoyo y protección más amplia a los que llegaron al territorio mexicano ${ }^{50}$.

Una vez que oficialmente se optó por acoger a los desplazados guatemaltecos, algunos de ellos pudieron adquirir la condición legal de refugiados, dando paso al establecimiento de más de 100campamentos reconocidos a lo largo de la línea fronteriza, en los cuales fueron implementados programas de vivienda, alimentación, educación y salud ${ }^{51}$. Para completar su ingreso se empleaban como asalariados, tanto en los lugares de asentamiento, como en sitios aledaños. Algunos de ellos, sembraban en terrenos alquilados por un pago en especie, en trabajo o, en las menos ocasiones, en dinero ${ }^{52}$.

Conforme pasaron los años -alrededor de quince-, esas necesidades combinadas con las condiciones de pobreza y marginación de Chiapas, transformaron las relaciones de

48 Carolina (habitante de Yalanhb'ojoch), entrevista con Fabiola Manyari López Bracamonte, Nentón, Guatemala, 03 de mayo 2017.

49 Con base en datos de entrevistas con la autora en la localidad de Nuevo Porvenir, México y la aldea de Yalanhb’ojoch, Guatemala (2015-2017).

50 Centro de Investigaciones y Estudios Superiores en Antropología Social, Los refugiados Guatemaltecos y lo Derechos Humanos, 8.

51 Hernández, y otros. La experiencia del refugio en Chiapas. Nuevas relaciones en la frontera sur mexicana, 56-57; Organización de los Estados Americanos, «Capítulo VII La Situación de los Refugiados y Desplazados en Guatemala y los Derechos Humanos,» en Cuarto informe sobre la situación de los derechos humanos en Guatemala (1a. ed.) (Washington: CIDH/OEA, 1993), 72.

52 Con base en datos de entrevistas con la autora en la localidad de Nuevo Porvenir, México y la aldea de Yalanhb’ojoch, Guatemala (2015-2017). 
apoyo que las familias mexicanas brindaron a las familias desplazadas. Estas llegaron a tornarse tensas principalmente por el acceso a la tierra para vivienda y labores productivas, que derivaron en formas de explotación a los refugiados ${ }^{53}$.

Disputas como esas junto con la condición de refugiado, generaron fronteras étnicas construidas a partir de las diferencias de nacionalidad y de acciones jurídicas, las cuales se alimentaron de las interacciones sociales desiguales ${ }^{54}$. Reconocerse o ser reconocido como refugiado se volvió un medio para acceder a las ayudas humanitarias, pero también se convirtió en una referencia identitaria, en muchas ocasiones discriminada por otros grupos sociales ${ }^{55}$.

Aunque el período de refugio fue un proceso heterogéneo, ese entorno requirió que las poblaciones refugiadas fortalecieran su capacidad de sobreponerse al terror por la violencia vivida, al dolor de las muertes, a las limitaciones en los campamentos, a las injusticias y a la discriminación ${ }^{56}$. Resultados de la investigación sobre el proceso de resiliencia comunitaria del pueblo chuj en esas décadas, demuestran que fue el conocimiento cultural, las capacidades sociales desprendidas de éste, así como la organización comunitaria lo que permitió mantenerlos con vida y motivó a reconstruir sus espacios sociales ${ }^{57}$.

53 Daniel Villafuerte, «Crisis rural, pobreza y hambre en Chiapas,» LiminaR Vol.13, n (2015): 13-28.

54 Rosa Hernández, Sur Profundo. Identidades Indígenas en la Frontera ChiapasGuatemala (México: Centro de Investigación y Estudios Superiores en Antropología Social, Comisión Nacional para el Desarrollo de los Pueblos Indígenas, 2012), 91-93. 55 Verónica Ruiz, Ser mexicano en Chiapas: identidad y ciudadanización entre los refugiados guatemaltecos en La Trinitaria (México: Instituto Nacional de Antropología e Historia. Colección Etnología y Antropología Social: Serie Logos. Instituto Nacional de Antropología e Historia, 2013), 255-258; Antonio Fabila, «Perspectiva histórica del refugio guatemalteco en México y los retos para su integración,» en La integración de los exrefugiados guatemaltecos en México, una experiencia con rostros múltiples, comp. Edith Kauffer (México: El Colegio de la Frontera Sur, San Cristóbal de Las Casas, 2002), 21- 28.

56 Claudia Anleu, Resiliencia la fuerza de la vida. El estudio sobre familiares de niñez desaparecida por el conflicto armado interno en Guatemala (Guatemala: Equipo de estudios comunitarios y acción psicosocial (ECAP), 2005), 63-65.

57 Fabiola López, «Resiliencia Comunitaria del pueblo Maya-Chuj: Conocimientos culturales, Capacidades Sociales y Estrategias organizativas» (Tesis de Doctorado, El 


\section{Acontecimientos en Guatemala durante el período del Refugio (1983-1996)}

Posterior al desplazamiento forzado, el contexto hostil en Guatemala se recrudeció por las políticas de control de la población y por las medidas de represión que se habían extendido hacia la población civil, particularmente en la zona rural. Luego del éxodo los mecanismos de control poblacional y militarización del país fueron mantenidos y perfeccionados por el ejército ${ }^{58}$.

Los militares intensificaron su presencia en el altiplano e implantaron una serie de figuras institucionales destinadas a concentrar a la población para su mayor vigilancia y sometimiento. El «Plan Nacional de Seguridad y Desarrollo (PNSD)», emitido por el Ejército después del golpe de Estado de 1982, se mantuvo vigente en la reorganización de la población desplazada internamente, y en aquellos que decidieron regresar poco tiempo después del desplazamiento forzado. Este plan articuló la estrategia de concentración de la población en "Aldeas Modelo» dentro de los llamados «Polos de Desarrollo» ${ }^{59}$, y de «las Patrullas de Autodefensa Civil (PAC)».

Las "Aldeas Modelo» eran coherentes con la búsqueda de transformar de forma rápida a la sociedad, a través del poder militar controlado por el Estado. Planteaban, agrupar a las personas indígenas en zonas específicas para apurarla modernización del país mediante la refuncionalización económica, social e ideológica.

colegio de la Frontera Sur, 2018), 143-147.

58 Hechos y políticas en Guatemala, Hechos y Políticas en Guatemala Enero-Marzo 1987 (Guatemala: Documento informativo anónimo, 1987), 3-6.

59 Los Polos de Desarrollo se refieren a una teoría de crecimiento económico que indica que el desarrollo como proceso polarizado, implica la formación de conglomerados y/o de cumbres de desarrollo. Con el tiempo dicha teoría amplió abarcando elementos normativos de intervención de política y de planificación (Hermansen, Tormod, «Polos y centros de desarrollo en el desarrollo nacional y regional: elementos de un marco teórico para un enfoque sintético,» Revista EURE-Revista de Estudios Urbano Regionales Vol. 4, $\mathrm{n}^{\circ} 10$ (1974): 55-96. 
En 1985 fue electo el primer civil en ocupar el cargo de Presidente de Guatemala, luego de 15 años de gobiernos militares. Desafortunadamente eso no significó un proceso de desmilitarización de la vida política de Guatemala ${ }^{60}$. Por el contrario, el mismo proceso electoral que había dado victoria a Vinicio Cerezo del partido Democracia Cristiana, fue orientado, dirigido y supervisado por los militares ${ }^{61}$. Por lo tanto, las decisiones sobre importantes aspectos de la política, la economía, la administración pública y la seguridad, seguían reservadas para ellos ${ }^{62}$.

Instituciones humanitarias y organizaciones laborales observaron con decepción cómo durante el primer año de gobierno democristiano (1985-1986), la represión se tornó altamente selectiva, tanto hacia las actividades insurgentes como hacia aquellas que involucraban cierto contenido de organización popular ${ }^{63}$. En diferentes ocasiones denunciaron que el número de asesinatos, secuestros políticos, y cadáveres que aparecían horriblemente torturados y mutilados ascendió significativamente (más de un centenar por mes), como también proliferó de nuevo el secuestro de familias campesinas ${ }^{64}$.

\section{Retorno y repatriación hacia Guatemala del pueblo chuj (1996-2016)}

60 Manz, Guatemala: Cambios en la comunidad, desplazamiento y repatriación, 263.

61 Durante esta década de 1980, se produjeron tres intentos de golpes de Estado, las cuales fueron reprimidos.

62 A través del Sistema Nacional de Coordinación Institucional para la Reconstrucción y el Desarrollo, se estableció la subordinación orgánica de todas las dependencias estatales y de cualquier organismo de tipo humanitario o independiente a autoridades militares. En 1986 Cerezo nombró como Ministro de Defensa a Alejandro Gramajo, quien colocó en importantes jefaturas militares a quienes eran cercanos a su afinidad contrainsurgente. (Hechos y Políticas en Guatemala, EneroMarzo 1987. Documento informativo anónimo, 1987).

63 Comisión de los Derechos Humanos de Guatemala, Informe presentado ante la subcomisión de prevención de discriminaciones y protección a las minorías (Guatemala: Naciones Unidas, 1989), 2.

64 Centro de Estudios de Guatemala, Guatemala: entre el dolor y la esperanza, coord. Luis Eduardo Martínez De León (España: Diputación Provincial de ValenciaCEDSALA- Universitat de Valencia, 1995), 77-81. 
Aunque la violencia y la militarización no habían cesado en Guatemala, el nuevo gobierno civil empezó a promover el regreso de las familias guatemaltecas al país. Fue así que en 1986 se creó la Comisión Nacional para la Atención de Repatriados, Refugiados y Desplazados (CEAR), integrada por los representantes de los Ministerios de Defensa, Relaciones Exteriores y Desarrollo, y del Comité de Reconstrucción Nacional, para coordinar la repatriación. Además en 1987, ACNUR abrió una oficina en la ciudad de Guatemala para facilitar la repatriación voluntaria de refugiados guatemaltecos ${ }^{65}$.

Quienes estaban en condición de refugio, se organizaron en un proceso que culminó con la formación de las Comisiones Permanentes de Refugiados Guatemaltecos en México (CCPP). El propósito de las Comisiones Permanentes, era negociar y promover el retorno organizado y colectivo de refugiados a Guatemala, e insertarse en grupos de la sociedad civil, que buscaban una mayor participación en las negociaciones de paz entre el gobierno y la $\mathrm{URNG}^{66}$. Un miembro representante de estas Comisiones recuerda las negociaciones de la siguiente manera:

\begin{abstract}
En este campamento nos mantuvimos varios años hasta que se organizó la Comisión Permanente, esta comisión se encargó hacer viajes para solicitar diálogos pacíficos con el gobierno de Guatemala donde plantearon la necesidad que hay entre los pueblos y pidieron que se compre tierra para las familias que no tienen tierras, de manera que puedan retornar organizadamente a su país de origen, así mismo regresar a su tierra de origen las personas que dejaron tierra en Guatemala, pero siempre con la condición de que el Estado garantice la vida de los habitantes, que se suprime la persecución del ejército sobre la población, que se reconozca el derecho de las personas y que no sean obligados al reclutamiento militar ${ }^{67}$.
\end{abstract}

65 ACNUR, La situación de los refugiados en el mundo 1997-1998. Un programa humanitario (España: Icaria, 1997), 100-102.

66 Instancia Mediadora-GRICAR El Proceso de Retorno de los Refugiados Guatemaltecos. Una Visión desde la Mesa de Negociación (Guatemala: Serviprensa, 1999), 56.

67 Entrevista colectiva, en discusión con la autora en Aldea de Yalanhb’ojoch, Guatemala, 15 febrero 2017). 
El carácter civil y pacífico que los grupos de refugiados tenían, tal como lo señala este relato, chocó con la militarización vigente en la mayor parte del territorio nacional, situación que desmotivaba a muchas de las personas que anhelaban desde años atrás el regreso a sus tierras ${ }^{68}$. Aunado a eso, a mediados la década de 1990 el gobierno de México abrió la posibilidad de integración para residir permanentemente en el país, y aunque al igual que el gobierno de Guatemala promovió el retorno ${ }^{69}$, implementó diferentes programas para la integración de refugiados ${ }^{70}$.

La disposición del estado mexicano en coordinación con el ACNUR y la COMAR fue reubicarles en los estados de Campeche y Quintana Roo, lugares donde se les dotaría de tierras, trabajos temporales y acompañamiento en sus trámites legales para la naturalización; sin embargo, no todas las familias accedieron a la reubicación, sobre todo por la lejanía con sus tierras de origen y las diferencias climáticas. Varias decidieron permanecer en La Trinitaria, lo que significó menor cobijo institucional y una lucha más complicada para acceder a espacios propios de tierra.

Quienes se quedaron en México enfrentaron problemas heterogéneos según su área de ubicación, aunque en común padecieron la unificación de los habitantes en un mismo asentamiento pese a su diversidad cultural, y las situaciones de marginación y discriminación que vivieron por la población mestiza y las instituciones mexicanas ${ }^{71}$.

68 Secretaría de La Paz, Entre pasado y olvido: Políticas de reconciliación en Guatemala 1996-2008 (Guatemala: Scuola Superiore Sant'Ann, 2009), 103.

69 Ricardo Falla, Negreaba de zopilotes, masacre y sobrevivencia, finca de San Francisco, Nentón (Guatemala: Asociación para el Avance de las Ciencias Sociales en Guatemala (AVANCSO), 2011), 196.

70 Oliver Levy, «La integración de los refugiados guatemaltecos en los estados de Campeche y Quintana Roo: una experiencia del proyecto de Unión EuropeaGobierno de México.» en La integración de los exrefugiados guatemaltecos en México, una experiencia con rostros múltiples, comp. Edith Kauffer (México: El Colegio de la Frontera Sur, 2002), 35-50.

71 Fabrizio Díaz, «Proceso de integración de los refugiados guatemaltecos al estado de Campeche,» en La integración de los exrefugiados guatemaltecos en México, una experiencia con rostros múltiples, comp. Edith Kauffer (México: El Colegio de la Frontera Sur, 2002), 75-81. 
Tuvimos que vivir con otras culturas, otros idiomas, con su costumbre diferente. Hay localidades que hasta hablan tres idiomas diferentes, pero así han tenido que tener acuerdo. Ya decidimos que no vamos a regresar a Guatemala porque nuestros hijos nacieron aquí, y pues tuvimos que luchar mucho para encontrar tierra, porque muchas veces los mexicanos no nos querían en ningún terreno, nadie nos apoyaba para lograr tener un espacio, ya ni la COMAR, ni el Comité Cristiano. Tampoco muchas veces nos quieren dar apoyo el gobierno porque no tenemos credencial de mexicanos. Ay fueron muchos años de lucha, de ir de acá para allá dejando nuestras cosechas, nuestra casita, hasta que al fin logramos comprar y tener papeles de que podemos estar, así es que estamos aquí ${ }^{72}$.

Quienes decidieron regresar a Guatemala también tuvieron experiencias diferenciadas. La repatriación de algunos fue procesada oficialmente y recibió asistencia de la CEAR, «Otros retornaron tal como se fueron, de manera anónima y sin asistencia de ningún tipo. Una vez en Guatemala, los repatriados individuales tendieron a dispersarse hacia localidades diversas» ${ }^{73}$. La diferenciación más importante, se debió al lugar donde cada quien regresó a vivir, pues no todos regresaron a la aldea o territorio de origen:

Nosotros suerte que pudimos regresar a nuestra tierra, recuperar nuestro lugar o el de nuestros padres. Mi hermano ya no quiso regresar, sólo yo con mi esposa y mis papás, sobre todo mi mamá era la que quería venir. Y volvimos y otra vez a construir nuestra casa. Porque aquí al menos tenemos tierra, en México mucha lucha y no se puede estar, pero ya no quisieron regresar porque tuvieron miedo quizás, no sé, o porque ya se acomodaron ahí. Pero nosotros suerte que volvimos y recuperamos nuestro lugar, otros de otras aldeas que también quisieron regresar pero ya estaba ocupado su lugar y tuvieron que ir, también otros que volvieron antes y que los Ejércitos los obligaban a otros espacios que no eran los suyos para poder vigilarlos ${ }^{74}$.

72 Lucía (habitante de Nuevo Porvenir), entrevista con Fabiola Manyari López Bracamonte, Chiapas, México 24 de agosto 2015.

73 Human Rights Watch/Americas, Human Rights in Guatemala, 73.

74 Gonzalo (habitante de Yalanhb'ojoch), entrevista con Fabiola Manyari López Bracamonte, Nentón, Guatemala, 28 de agosto 2015. 
Como puede apreciarse en ambos relatos, las poblaciones chuj reasentadas en ambos lados de la frontera, debieron enfrentarse a la reconstrucción de sus territorios, de sus hogares y de sus familias, todo dentro de un contexto sociopolítico que no atendía sus demandas históricas ni integraba sus perspectivas culturales. Como consecuencia, las relaciones desiguales provenientes de un Estado modernizador a costa y en contra de los pueblos originarios permanecieron vigentes.

Para el pueblo chuj que regresó a Guatemala, esas relaciones desiguales se mantuvieron vigentes por ejemplo en el conflicto por la preservación de los recursos naturales ${ }^{75}$, pues el Estado siguió imponiendo la construcción de la carretera que parte de la Franja Transversal del Norte que atraviesa su territorio ${ }^{76}$, disputa que desde 1950 ha formado parte de la intención modernizadora del Estado guatemalteco:

[...] Ya no estuvo tanto miedo cuando venimos aquí [al volver del refugio]. Estuvieron las patrullas pero ya hace mucho tiempo, ya casi se calmó, ya se terminó; pero ahorita hay otro problema: las empresas de otro país, de Israel dicen, se están entrando aquí otra vez, aquí por valle de Guatemala, por Centroamérica. Pues esos también, pues están trabajando por la carretera transversal del norte. Aquí están, aquí abajo por una aldea que se llama Ixquisis; ahí están contaminando a la gente, están contaminando el agua ahí ${ }^{77}$.

75 Cecilia Mérida y Wolfgang Krenmayr, Asamblea departamental por la defensa de los recursos naturales renovables y no renovables en Huehuetenango. Sistematización de experiencias 2008-2009. Tejiendo entre los pueblos la defensa del territorio (Guatemala: Serviprensa, 2010), 37-41.

76 Aldeas de Nentón, Huehuetenango organizada ha logrado recuperar la Laguna Brava de extranjeros que han querido privatizarla, deteniendo la construcción de la Vía carretera de la Franja Transversal del Norte debido a que la empresa Israelí Solel Boneh irrumpió en las comunidades sin consulta previa, además de utilizar fuentes de agua para mantenimiento de maquinarias y no negociar un pago justo por los inmuebles afectados por la construcción de la carretera (Pérez, y otros, Energía renovable en comunidades campesinas de los municipios de Chaal, Panzós, Cahabón, Cubulco, Tacaná, San Marcos, San Pedro, Chajul y Nentón (Guatemala: Universidad de San Carlos de Guatemala, Dirección General de Investigación, Informe final de investigación 2011, Programa Universitario de Investigación en Energía, DIGIEscuela de Historia, USAC-Fundación Solar, 2012).

77 Alberto (habitante de Yalanhb'ojoch), entrevista por Fabiola Manyari López Bracamonte, julio de 2016. 
Para el pueblo chuj, en México el contexto hostil también siguió vigente.

Principalmente a partir de la negación y discriminación de su esencia cultural enraizada en Guatemala, estrategia nacionalista de integración iniciada desde el siglo pasado. Experiencias como la anterior describen esta situación:

Yo no aprendí muy bien Chuj, no me quisieron enseñar porque luego en la escuela los otros niños se burlaban porque que somos indios, y los mismos maestros te regañan que sólo se hable en Castilla [español], y si no lo hablas no aprendes nada en la Escuela y todos te miran mal. Así igual lo vivieron mis papás y no quisieron que sufriera eso. Recién es que ahora quiero aprender, porque ellos [mis papás] están hablando en Chuj y yo no entiendo, y pienso que sería bonito entender lo que están diciendo ${ }^{78}$.

Pese a esas realidades, el pueblo chuj mantiene la esperanza de mejorar sus condiciones de vida, incluyendo la reivindicación de su vida cultural, ahora como pueblo transfronterizo. Las formas y las directrices para lograr esta renovación son aún un proyecto en construcción, para el que las experiencias del desplazamiento y el refugio son fundamentales. Yakin quien vivió el desplazamiento a los 5 años de edad y que posteriormente decidió formar su familia en México lo planteó de esta manera:

Muchos dicen que nosotros somos débil [es], pero cuando estamos hablando de esto me doy cuenta que en realidad no somos débiles, ¿quién no se va a huir de una guerra?, enfrentamos al Ejército, al demonio, podemos decir así porque esos están endemoniados, porque cualquier persona que vean no le van a tener lástima. Luego llegar a México y aunque estamos un poco contento que no nos mató el Ejército, también sufrimos por mantener a nuestras familias pero salimos adelante. Así hemos seguido haciendo el camino, así hay que decirles a los jóvenes, a los chamacos ${ }^{79}$.

78 Diana (habitante de Nuevo Loma Linda), entrevista por Fabiola Manyari López Bracamonte, Chiapas, México, 12 de febrero 2017.

79 Yakin (habitante de Nuevo Porvenir), entrevista por Fabiola Manyari López Bracamonte, Chiapas, México, 18 de noviembre 2018. 
Kixtup, joven hijo de Yakin quien escuchaba la entrevista con su padre cerró la conversación con la siguiente reflexión:

\begin{abstract}
A veces es triste cuando escucho a mis padres o a mis abuelos contar eso que sufrieron, y me pregunto ¿no será mejor olvidarlo, para qué recordar eso?, pero luego me doy cuenta que eso que cuentan es parte de su lucha, porque han luchado mucho y siguen luchando, ahora yo más. Darme cuenta que como jóvenes a veces no valoramos eso, pero que su historia nos ayuda a entender mejor quiénes somos, que somos un pueblo fuerte, porque tal vez otros hubieran muerto. Ahora es pensar cómo le vamos a hacer para que podamos valorar eso, y explicárselos a los otros jóvenes que no les importa, que su idea es sólo irse a otro lado para ganar dinero. [...] Osea ser chuj es todo eso, también los que viven en Guatemala, porque estamos divididos sólo por una frontera de los gobiernos, pero somos parte del mismo pueblo ${ }^{80}$.
\end{abstract}

La reflexión intergeneracional de los fragmentos anteriores, posibilita encontrar nuevas formas de reivindicar su duro pasado reconociendo el valor y la dignidad de los habitantes chuj frente a los embates históricos a su existencia. Aspecto que resulta de gran importancia en este tipo de investigaciones que buscan una reconstrucción integradora entre la memoria histórica y la colectiva.

\title{
Conclusiones
}

El conflicto armado y la violencia de Estado que llevó al desplazamiento forzado de pueblos mayas en Guatemala como el chuj, se integró por la confluencia de diferentes intereses globales y condiciones locales que al reconstruirse históricamente, permiten describir la cualidad de las interacciones entre ambas escalas, así como clarificar las tensiones en la dinámica social que posibilitaron las catastróficas consecuencias.

A partir de esa reconstrucción se evidencian particularidades del conflicto como consecuencia de la Guerra Fría en Guatemala, caracterizadas por la legitimación de la

80 Kixtup (habitante de Nuevo Porvenir), entrevista por Fabiola Manyari López Bracamonte, Chiapas, México, 18 de noviembre 2018. 
violencia mediante la construcción de una "otredad enemiga», que incluyó a grupos considerados contrarios a las lógicas de modernización imperantes: movimientos insurgentes y pueblos originarios. Siendo estos últimos las mayores víctimas dada la necesidad del Estado de erosionar sus componentes étnicos «inútiles» para las lógicas capitalistas.

En ese contexto el pueblo chuj fue uno de los pueblos violentados física y simbólicamente, y aunque logró sobrevivir al exterminio, tuvo que asumir cambios en sus actividades cotidianas, sus prácticas culturales y organizativas. Al igual que otros pueblos desplazados fueron testigos de la destrucción de su mundo social y material, sin embargo su esperanza por la vida, y sus capacidades organizativas, les ayudaron a buscar alternativas de sobrevivencia y resistencia durante el refugio, y posteriormente de reconstrucción en el retorno a Guatemala o en la integración a México.

Como se realizó en este artículo, complementar la revisión histórica con datos provenientes de la memoria colectiva, evoca una postura sensible para adentrarse a estas temáticas de conflicto armado y genocidio, donde la constante es el sufrimiento humano. Además, los testimonios y relatos aportan datos concretos, que aunque no necesariamente son objetivos, permiten reinterpretar los sucesos desde la subjetividad de las percepciones y las emociones. Como sucedió en esta investigación, vincular a los afectados con datos históricos para incentivar los recuerdos de reconstrucción de los hechos, propicia un diálogo reflexivo que nutre a su vez la memoria de los pueblos.

Es decir, este acercamiento entre historia y memoria, además de abonar a las discusiones provenientes de las ciencias sociales, promueve un ejercicio de participaciónreflexión en las víctimas que les alerta sobre la necesidad de mantenerse al tanto de las pugnas en el territorio, de sus orígenes, sus escalas y los intereses de los involucrados. Les invita a reconocer que las disputas territoriales, entre ellas los conflictos bélicos, son procesos gestados tiempo atrás por diferentes agentes. Por lo tanto su solución requiere la 
atención de esas tensiones de origen y sus participantes, ya que de no hacerlo los conflictos permanecerán latentes.

Finalmente, contrastar los datos históricos con datos de la memoria colectiva, permite reconocer el impacto de estos acontecimientos en la psiquis de las personas y en sus dinámicas colectivas y culturales, lo que aporta datos valiosos en la promoción de la recuperación frente el horror vivido. Es decir, la reelaboración del pasado y de su historia como pueblo con fines de renovación.

\section{Bibliografía}

\section{Fuentes primarias}

ACNUR (Alto Comisionado de las Naciones Unidas para los Refugiados), La situación de los refugiados en el mundo 19971998. Un programa humanitario. España: Icaria, 1997.

Anleu, Claudia. Resiliencia la fuerza de la vida. El estudio sobre familiares de niñez desaparecida por el conflicto armado interno en Guatemala. Guatemala: Equipo de estudios comunitarios y acción psicosocial (ECAP), 2005.

Arzobispado de Guatemala. Informe del Proyecto Interdiocesano: Recuperación de la Memoria Histórica. TOMO II. Guatemala: Oficina de Derechos Humanos del Arzobispado de Guatemala ODHAG, 1998.

Arzobispado de Guatemala y Oficina de Derechos Humanos. «Versión popular del informe REMHI». Guatemala: Nunca Más. Oficina de Derechos Humanos del Arzobispado de Guatemala, 2000.

CEPAL (Comisión Económica para América Latina). Notas sobre la evolución del desarrollo social del istmo centroamericano hasta 1980. Naciones Unidas. Consejo Económico y Social. Distribución limitada E/CEPAL/MEX/1982/L.26/Rev.1.(1982). http://repositorio.cepal.org/bitstream/handle/11362/26485/ S8200536_es.pdf?sequence=1\&isAllowed=y [29-03-2017.

Centro de Investigaciones y Estudios Superiores en Antropología Social. Los refugiados Guatemaltecos y los Derechos Humanos. 
Cuaderno para Refugiados Guatemaltecos. México: Gobierno del Estado de Chiapas/Consejo Estatal de Fomento a la Investigación y Difusión de la Cultura/DIF-Chiapas/Instituto Chiapaneco de Cultura, 1991.

Centro de Estudios de Guatemala. Guatemala: entre el dolor y la esperanza, coordinado por Luis Eduardo Martínez De León. España: Diputación Provincial de Valencia-CEDSALAUniversitat de Valencia, 1995.

Centro de Noticias de Guatemala. Guatemala 1986-1987. Crisis del 1er. año de Gobierno de Vinicio Cerezo. Estados Unidos de América: documento anónimo, 1987.

CEH. Informe de la Comisión para el Esclarecimiento Histórico. TOMO III: Las violaciones de los derechos humanos y los hechos de violencia. Guatemala: Oficina de Servicios para Proyectos de las Naciones Unidas (UNOPS), 1999.

CDHG. Testimonios sobre las Patrullas de Autodefensa Civil (PAC) en Huehuetenango. Guatemala: Comisión de Derechos Humanos de Guatemala, 1985.

Contexto Histórico de la Aldea de Yalanhb'ojoch.Contexto Histórico de la Aldea de Yalanhb'ojoch, del municipio de Nentón, Departamento de Huehuetenango. Guatemala: Programa Nacional de Resarcimiento, Unidad de Aplicación de Medidas, 2009 .

Comisión delos Derechos Humanos de Guatemala.Informepresentado ante la subcomisión de prevención de discriminaciones $y$ protección a las minorías. Guatemala: Naciones Unidas, 1989.

Hechos y Políticas en Guatemala Enero-Marzo 1987. Documento informativo anónimo, 1987.

Historia del pueblo de Guatemala. Documento informativo de distribución anónima, 1983. Human Rights Watch/Americas. Human Rights in Guatemala Durind President de León Carpio's First Year, traducido por Luis E. Bossio. Guatemala: Luna y Sol editorial, 1994. 
Instancia Mediadora-GRICAR.El Proceso de Retorno de los Refugiados Guatemaltecos. Una Visión desde la Mesa de Negociación. Guatemala: Serviprensa, 1999.

López, Fabiola. «Resiliencia Comunitaria del pueblo Maya-Chuj: Conocimientos culturales, Capacidades Sociales y Estrategias organizativas.» Tesis de Doctorado, El colegio de la Frontera Sur, 2018.

MajawilQ'ij. El Nuevo Amanecer. Guatemala: Folleto informativo de la Coordinación maya MajawilQ’ij"', 1992.

Manz, Beatriz.Guatemala:Cambiosen la comunidad, desplazamiento y repatriación. México: Escuela de Antropología Wellesley College, Centro de Estudios Internacionales Harvard University. Traducción libre al español de la primera versión del documento en inglés; no revisada por la autora. Praxis, 1986.

Mérida, Cecilia, y Wolfgang Krenmayr. Asamblea de departamental por la defensa de los recursos naturales renovables y no renovables en Huehuetenango. Sistematización de experiencias 2008-2009. Tejiendo entre los pueblos la defensa del territorio. Guatemala: Serviprensa, 2010.

Organización de los Estados Americanos. «Capítulo VII La Situación de los Refugiados y Desplazados en Guatemala y los Derechos Humanos.» En Cuarto informe sobre la situación de los derechos humanos en Guatemala, 1a. ed. Washington: CIDH/ OEA, 1993.

Pérez, María, María Morales, y Amarilis Enriquez. Energía renovable en comunidades campesinas de los municipios de Chaal, Panzós, Cahabón, Cubulco, Tacaná, San Marcos, San Pedro, Chajul y Nentón. Guatemala: Universidad de San Carlos de Guatemala, Dirección General de Investigación, Informe final de investigación 2011, Programa Universitario de Investigación en Energía, DIGI-Escuela de Historia, USAC-Fundación Solar, 2012.

Reflexión Cristiana en Guatemala. Derechos de los pobres, derechos de Dios. Guatemala: grupo de Reflexión Cristiana en Guatemala, noviembre 1990. 
Secretaría de La Paz. Entre pasado y olvido: Políticas de reconciliación en Guatemala 1996-2008. Guatemala: Scuola Superiore Sant'Ann, 2009.

Stoll, David. Between Two Armies in the Ixil Towns of Guatemala. Nueva York: Columbia University Press, 1993.

Universidad de San Carlos d e Guatemala. Guatemala: Proceso de paz y perspectivas. Guatemala: Escuela de historia. S.f.

\section{Fuentes secundarias}

Aguilera, Gabriel, y Jorge Imery. Dialéctica del terror en Guatemala. CostaRica: Editorial Universitaria Centroamericana, 1981.

Armony, Ariel. «Trasnacionalización del Caribe: catalizador de la proyección soviética en América Latina.» En Espejos de la Guerra Fría: México, América Central y el Caribe, coordinado por Daniela Spencer. México: Centro de Investigaciones y Estudios Superiores en Antropología Social. Porrúa, Distrito Federal, 2004.

Balsells, Edgar. Olvido o memoria: el dilema de la sociedad guatemalteca. 2da ed. Guatemala: FLACSO, 2001.

Ball, Patrick, Paul Kobrak, y Herbert Spirer. Violencia institucional en Guatemala, 1960 a 1996: una reflexión cuantitativa. American Association for the Advancement of Science (AAAS) Science and Human Rights Program. Estados Unidos: Centro Internacional para Investigaciones en Derechos Humanos (CIIDH), 1999.

Castañeda, César. Lucha por la tierra, retornados y medio ambiente en Huehuetenango. Guatemala: FLACSO, 1998.

Centro de Estudios Integrados de Desarrollo Comunal. Guatemala polos de desarrollo. El caso de la desestructuración de las comunidades indígenas. México: Praxis, 1988.

Shelton, Davis. "Introduccion: Sowing the Seeds of Violence.» EnHarverst of violence. The Maya Indians and the Guatemala Crisis, editado por Robert M. Carmarck, 3-16. United States of América: University of Oklahoma Press, 1988. 
Díaz, Fabrizio. «Proceso de integración de los refugiados guatemaltecos al estado de Campeche.» En La integración de los exrefugiadosguetemaltecos en México, una experiencia con rostros múltiples, compilado por Edith Kauffer. 75-81. México: El Colegio de la Frontera Sur, San Cristóbal de Las Casas, 2002.

Fabila, Antonio. «Perspectiva histórica del refugio guatemalteco en México y los retos para su integración.» En La integración de los exrefugiados guatemaltecos en México, una experiencia con rostros múltiples, compilado por Edith Kauffer, 21-28. México: El Colegio de la Frontera Sur, 2002.

Falla, Ricardo. Negreaba de zopilotes, masacre y sobrevivencia, finca de San Francisco, Nentón. Guatemala: Asociación para el Avance de las Ciencias Sociales en Guatemala (AVANCSO), 2011.

Figueroa, Carlos. «Genocidio y terrorismo de Estado en Guatemala (1954-1996): una interpretación.» En Guatemala: historia reciente (1954-1996). Tomo I: proceso político y antagonismo social, 68-89. Guatemala: Facultad Latinoamericana de Ciencias Sociales, 2012.

Gramajo, Hector. La difícil transición política en Guatemala. De la guerra... a la guerra. Guatemala: Fondo de Cultura Editorial, 1995.

Gurriaran, Javier.La resistencia en Guatemala. México: Nuestro Tiempo, 1989.

Gutiérrez, Estela, y Paul Kobrak. Los linchamientos postconflicto y Violencia colectiva en Huehuetenango Guatemala. Guatemala: CEDGOF, 2001.

Hermansen, Tormod. «Polos y centros de desarrollo en el desarrollo nacional y regional: elementos de un marco teórico para un enfoque sintético.» Revista EURE-Revista de Estudios Urbano Regionales Vol. 4, $\mathrm{n}^{\circ} 10$ (1974): 55-96.

Hernández, Rosa. Sur Profundo. Identidades Indígenas en la Frontera Chiapas-Guatemala. México: Centro de Investigación 
y Estudios Superiores en Antropología Social, Comisión Nacional para el Desarrollo de los Pueblos Indígenas, 2012.

Hernández, Rosalva, Norma Nava, Carlos Flores, y José Escalona. La experiencia del refugio en Chiapas. Nuevas relaciones en la frontera sur mexicana. México: Academia Mexicana de Derechos Humanos A.C., Centro de Investigaciones y Estudios Superiores en Antropología Social, Consejería en Proyectos para Refugiados Latinoamericanos, OXFAM, United Nations Research Institute for Social Development, Copilco Universidad, 1993.

Katz, Friedrich. "La guerra fría en América Latina.» En Espejos de la guerra fría: México, América Central y el Caribe, coordinado por Daniela Spencer, 11-30. México: Centro de Investigaciones y Estudios Superiores en Antropología Social. Porrúa, 2004.

Kauffer, Edith. «De la frontera política a las fronteras étnicas Refugiados guatemaltecos en México.» Frontera Norte Vol. 17, $\mathrm{n}^{\circ} 34$ (2005): 7-36.

Kobrak, Paul. Huehuetenango: historia de una guerra. Guatemala: Centro de Estudios y Documentación de la Frontera Occidental de Guatemala (CEDFOG), 2003.

Lazo, Francisco. «La estrategia norteamericana y la geopolítica de América Central en América Central y el Caribe.» En Zona de Conflictos y problemas geopolíticos. México: Cuaderno de Trabajo No. 8. Centro de Investigación y Trabajo social, 1986, $23-42$.

Leal, Francisco. «La Doctrina de Seguridad Nacional en América Latina: materialización de la guerra fría en América del Sur.» Revista de Estudios Sociales, n 15 (2003): 74-87.

Levy, Oliver.«La integración de los refugiados guatemaltecos en los estados de Campeche y Quintana Roo: una experiencia del proyecto de Unión Europea-Gobierno de México.» En La integración de los exrefugiados guatemaltecos en México, una experiencia con rostros múltiples, compilado por Edith Kauffer, 35-50. México: El Colegio de la Frontera Sur, 2002. 
Mc Allister, Carlota. «Mercados rurales, almas revolucionarias y mujeres rebeldes en la Guatemala de guerra fría.» En Espejos de la guerra fría: México, América Central y el Caribe, coordinado por Daniela Spencer. México: Centro de Investigaciones y Estudios Superiores en Antropología Social, Porrúa, 2004.

Palencia-Ferrer, Sergio. «Rebelión social y contrainsurgencia en Guatemala, 1981-1983. Conformación estatal y potencialidad revolucionaria.» Liminar, Estudios Sociales y Humanísticos Vol. XII, nº 1 (2014): 161-176, 2014.

Perkič-Krempl, Sonja. «El Derecho a Saber.» Tesis de Maestría, Universidad Rafael Landivar, 2006.

Pita, Alexandra, Enrique Coraza, y Karla Amador. «La Doctrina de Seguridad Nacional en América Latina: el concepto de "enemigo"." En La Guerra Fría y las Américas, coordinado por Avital Bloch y María del Rosario Rodríguez, 16-34. México: Universidad de Colima. Centro Universitario de Investigaciones Sociales. Universidad Michoacana de San Nicolás de Hidalgo. Instituto de Investigaciones Históricas, 2013.

Ruiz, Verónica. Ser mexicanoen Chiapas:identidadyciudadanización entre los refugiados guatemaltecos en La Trinitaria. México: Instituto Nacional de Antropología e Historia. Colección Etnología y Antropología Social: Serie Logos. Instituto Nacional de Antropología e Historia, 2013.

Solano, Luis. Contextualización Histórica de la Franja Transversal del Norte (FTN). Guatemala: Centro de Estudios y Documentación de la Frontera Occidental de Guatemala CEDFOG, 2012.

Sohr, Raúl. Centroamérica en guerra. Las fuerzas armadas en de Centroamérica y México. México: Alianza Editorial Mexicana, 1989.

Tejada, Mario. Historia social del norte de Huehuetenango. Guatemala: Magna Torres, 2002. 
Torres, Edelberto. «Guatemala: desarrollo, democracia y los acuerdos de paz.» Revista Centroamericana de Ciencias Vol. $3, \mathrm{n}^{\mathrm{o}} 2$ (2006): $11-48$.

Villafuerte, Daniel. «Crisis rural, pobreza y hambre en Chiapas.» LiminaR Vol.13, $\mathrm{n}^{\circ} 1$ (2015): 13-28.

\section{Citar este artículo}

López Bracamonente, Fabiola Manyari. "Conflicto armado en Guatemala: reconstrucción histórica y memoria colectiva del pueblo maya chuj.» Historia Y MEMORIA, n 22 (2021): 323-357.DOI:https:/doi.org/10.19053/20275137.n22.2021.10791. 